\title{
Endobronchial polyp in an asthmatic subject
}

\author{
DJ SHALE, DJ LANE, CWS FISHER, MS DUNNILL
}

From the Osler Chest Unit, Churchill Hospital, and John Radcliffe Hospital, Oxford

There is an association between nasal polyposis, nonatopic asthma, and sensitivity to aspirin or non-steroidal anti-inflammatory agents. ${ }^{1}$ Although there are structural and functional similarities between nasal and bronchial mucosa similar polyps have not been described in the lower respiratory tract of asthmatics.

\section{Case report}

The patient, a 51-year-old man who worked as a post office technician, complained of progressive breathlessness during four weeks after return to work after a cholecystectomy. He was exposed to vapour from soldering with a rosin-containing flux in a multicore-type solder. He was producing mucopurulent sputum, but denied any haemoptysis or wheezing. He had a 30-year history of stable non-atopic asthma. Four years earlier, during an exacerbation of his asthma, a peripheral blood eosinophilia had been found and lung biopsy had showed an alveolar and interstitial infiltrate with eosinophils and features of an eosinophilic vasculitis. The underlying aetiology was not defined and he improved with corticosteroid treatment, which was eventually withdrawn. He had required surgical treatment for nasal polyposis and sinusitis. There was no significant family history and no history of cigarette smoking. He denied any allergies and gave no history of hypersensitivity to aspirin, tartrazine, or non-steroidal anti-inflammatory agents. On examination abnormal signs were confined to the respiratory system, where there was evidence of collapse of the left lower lobe. On the right side only rhonchi were heard. A chest radiograph confirmed major collapse of the left lower lobe and an associated pleural effusion. The haemoglobin concentration was $16.9 \mathrm{~g} / \mathrm{dl}$ and the white blood cell count $20.3 \times$ $10 \% / 1,46 \%$ of cells being eosinophils (absolute count $9.3 \times$ $10 \% / 1)$. There were no responses in skin-prick tests for common allergens, including Aspergillus fumigatus. Serum precipitins for aspergillus were not detected. There was a negative result in the antinuclear factor test but rheumatoid factor was present (1/64). Serum complement (C3 and C4), IgG, IgM, IgA, and IgE levels were normal. Neither bacteria nor fungi could be cultured from sputum but cytological examination showed numerous eosinophils. Pleural aspiration yielded serous fluid with $52 \mathrm{~g} / 1$ of protein and no growth on culture; over $90 \%$ of the cells present were eosinophils.

At bronchoscopy a polyp was found to be obstructing the left lower-lobe bronchus. The polyp was removed in a piecemeal fashion and treatment with prednisolone $60 \mathrm{mg}$ daily was started. There was rapid improvement with re-

Address for reprint requests: Dr DJ Shale, Osler Chest Unit, Churchill Hospital, Oxford OX3 7LJ.

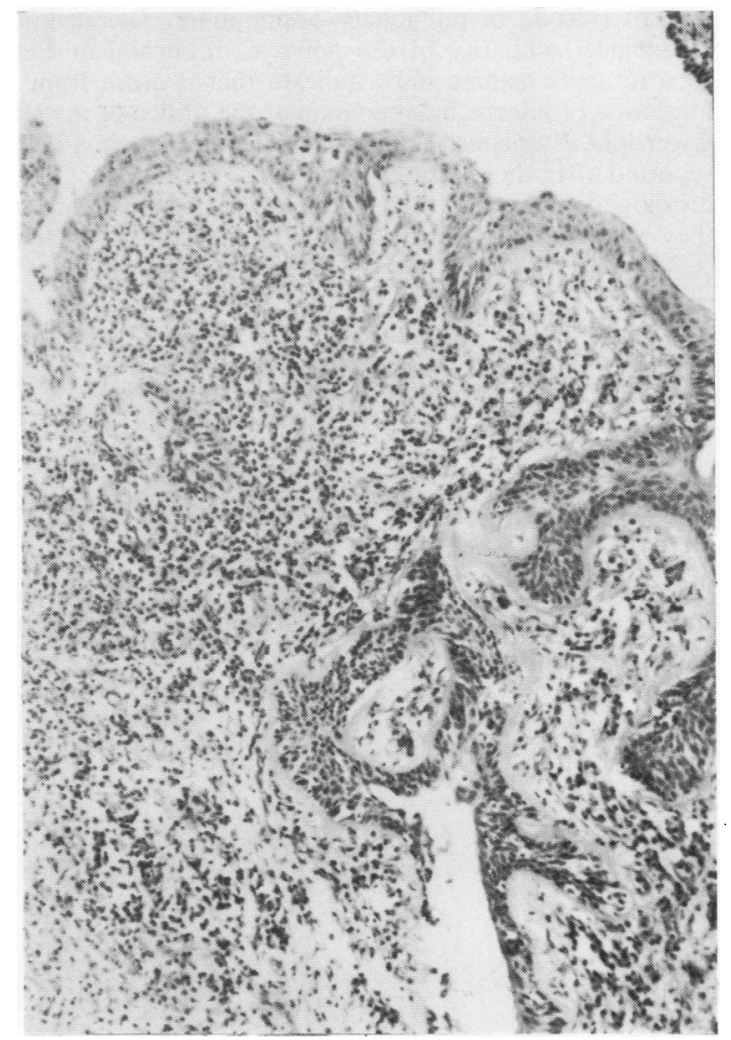

Photomicrograph of the endobronchial polyp showing squamous metaplasia of the overlying epithelium, some basement membrane thickening, and intense eosinophil leucocytic infiltration of the underlying stroma.

(Haematoxylin and eosin, $\times 262$.)

expansion of the left lung. Histological examination showed an oedematous fibrous stroma, which was heavily infiltrated with eosinophil leucocytes and lymphocytes. Typical features of asthmatic bronchial mucosa were noted, with variation in the covering epithelium between a simple stratified type and normal respiratory epithelium. There was some focal thickening of the basement membrane, which was not thought to be due to cross-cutting (fig).

After cessation of oral steroid treatment the patient was challenged with solder fumes. His peak expiratory flow rate (PEFR) was monitored for 24 hours before and after a two-hour peroid of soldering. There was no effect on 
PEFR values. Regular PEFR measurements by the patient at work during periods of exposure and non-exposure to solder vapour showed no deterioration of airways function related to soldering.

\section{Discussion}

We report the unique case of an endobronchial polyp in a non-atopic asthmatic patient with previous nasal polyposis and an episode of pulmonary eosinophilia. The striking histological similarity of the polyp to bronchial mucosa seen in acute asthma must indicate that it arose from a local area of intense inflammation at the orifice of the left lower-lobe bronchus. Endobronchial polyposis has been reported after thermal injury to the respiratory tract. The histological nature of these polyps was not reported but they resolved with corticosterid treatment. ${ }^{2}$ Our patient was exposed over a four-week period, in confined conditions, to hot vapours produced during soldering. Although $\stackrel{x}{.}$ non-specific irritation due to this may have contributed to $\overrightarrow{\bar{F}}$ the formation of the polyp, there was no evidence that occupational asthma played a part. We cannot rule out a link with the previous eosinophilic vasculitis but this had been in clinical remission for four years.

References

${ }^{1}$ Morris-Owen $\mathrm{R}$. The natural history of asthma. In: Lane DJ, ed. Tutorials in postgraduate medicine: respiratory. disease. London: Heinemann, 1976:ch 12.

${ }^{2}$ Adams C, Moisan T, Chandrasekhar AJ, Warpeha R, Endobronchial polyposis secondary to thermal inhala- $\tilde{C}_{\infty}$ tional injury. Chest 1979;75:643-5. 